Ann. Biol. anim. Bioch. Biophys., I973, 13 (2), I77-I85.

\title{
EFFETS VARIABLES DE L'OCYTOCINE ET DES KININES SUR LA PRESSION INTRAMAMMAIRE D'UNE BREBIS EN LACTATION
}

\author{
G. PEETERS et A. HOUVENAGHEL \\ Laboratoive de Physiologie, \\ Faculté de Médecine vétérinaire de l'Université, \\ Gand (Belgique)
}

\section{RÉSUMÉ}

La pression intramammaire a été enregistrée au cours de nombreuses expériences effectuées sur des brebis et des chèvres en lactation recevant de l'ocytocine, de la bradykinine et de la kallikréine administrées directement dans l'artère mammaire. Dans une expérience effectuée sur une brebis anesthésiée, les 3 substances ont provoqué des augmentations mais également des diminutions de la pression intramammaire. Les effets hypertensifs sont attribués à la contraction des cellules myoépithéliales situées autour des alvéoles, provoquant ainsi l'expulsion du lait. Les effets hypotensifs seraient dus au contraire à la contraction des cellules myoépithéliales situées longitudinalement par rapport aux canalicules et canaux mammaires, ce qui entraînerait le raccourcissement et la dilatation de ces derniers. Pendant la seconde moitié de l'expérience, le myoépithélium des canaux est apparu plus sensible à l'action des substances étudiées que le myoépithélium des alvéoles. La dose seuil pour l'obtention de la dilatation des canaux était alors plus faible, la réponse se manifestant plus tôt et se maintenant plus longtemps que celle conduisant à la contraction des alvéoles.

\section{INTRODUCTION}

Dans la glande mammaire on distingue 2 sortes de cellules myoépithéliales : des cellules en forme d'étoile formant un réseau compliqué autour des alvéoles, et des cellules fusiformes situées en direction longitudinale par rapport à la surface externe des canaux galactophores (RICHARDSON, I949; LINZELL, I952).

En se basant sur leurs aspects morphologiques et sur les résultats obtenus lors d'enregistrements de la pression intramammaire et de l'écoulement du lait chez la Vache et la Truie (WhITTLESTONE, I950), on admet généralement que l'hormone de l'éjection du lait provoque la contraction des cellules myoépithéliales situées autour des alvéoles et des canaux galactophores. Il en résulte simultanément une compression des alvéoles, un raccourcissement et une dilatation des canaux. La 
baisse de la résistance des canaux permet ainsi l'afflux rapide du lait dans les larges canaux et les citernes.

Des expériences sur la pression intramammaire chez des femmes (ZAKS et MAZHBITZ, I965) et des observations microscopiques in vivo dela glande mammaire de petits animaux (LINZEII, I955 ; LEVITSKAYA, I955) ont mené aux mêmes conclusions.

Au cours des dernières années nous avons étudié l'influence de l'ocytocine, des kinines du plasma et de la kallikréine sur la pression intramammaire chez un grand nombre de brebis et de chèvres en lactation (PEETERs, Houvenaghel, Verbeke; et VAN SichEM-REYNAERT, I972). La bradykinine injectée dans 1'artère de la glande augmente la pression intramammaire en agissant probablement sur les cellules myoépithéliales, mais son action est environ $5^{\circ}$ fois plus faible que celle de l'ocytocine. La kallikréine administrée par la même voie libère des kinines à partir des $\alpha$-globulines du plasma et provoque également l'éjection du lait. En outre, les kinines possèdent une action vasodilatatrice importante dans la glande mammaire. Dans une de ces expériences des effets remarquables sur la pression intramammaire ont été obtenus qui plaident en faveur du rôle distinct mentionné ci-dessus des cellules en étoile et des cellules fusiformes. Cette expérience est mentionnée en détail dans cette communication.

\section{TECHNIQUES EXPÉRIMENTALES ETT MATÉRIEL UTILISÉ}

Cinquante-sept expériences ont été effectuées sur 30 brebis et 17 chèvres en lactation. Les effets décrits ci-dessous n'ont été observés qu'au cours d'une seule expérience sur une brebis de race Texel. Celle-ci est un animal primipare (poids $36 \mathrm{~kg}$ ); elle a été étudiée quelques jours après la mise bas (production de lait : $800 \mathrm{ml} / \mathrm{j}$ ). Son pis est normal avec des cavités citernales peu développées. Le soir précédant l'expérience, la brebis a été mise à jeun et le matin de l'expérience elle ne fut pas traite. L'atropine (10 $\mathrm{mg}$ ) et la prométhazine ( ${ }^{1}$ ) (50 $\mathrm{mg}$ ) ont été administrées avant l'expérience, puis l'animal a été fixé sur le dos et anesthésié avec le mélange protoxyde d'azote-oxygène $(75 / 25)$ et halothane. L'artère pudenda externa a été dégagée et la pression intramammaire enregistrée dans la glande ipsi laterale. Les substances étudiées ont été administrées par injection rapide dans l'artère à l'aide d'un tube de polyéthylène ayant une longueur de $30 \mathrm{~cm}$ et un diamètre interne de $0,7 \mathrm{~mm}$. La surface de la glande a été recouverte par un bandage adhésif dans le but de rendre l'enregistrement de la pression plus sensible. Enfin, un cathéter introduit dans le trayon reliait, par un tube de polyéthylène rempli d'une solution de citrate à $\mathbf{~ p}$. Ioo, les cavités mammaires à un traducteur de pression Statham. La pression intramammaire a été enregistrée par un appareil Rohde et Schwarz. Les solutions ont. été injectées par intervalles de 5 minutes.

Nous avons utilisé l'ocytocine ( ${ }^{2}$ ) et la bradykinine synthétiques (Sandoz, Bâle). La kallikréine était une préparation commerciale $\left(^{3}\right)$ isolée de pancréas et de glandes salivaires de porc. Les 3 substances ont été dissoutes aux concentrations suivantes dans du $\mathrm{NaCl} 9 \mathrm{p}$. I ooo : bradykinine $100 \mathrm{ng} / \mathrm{ml}$, ocytocine 0,2 et $\mathrm{I} \mathrm{mU} / \mathrm{ml}$, kallikréine 0,2 et $\mathrm{I} \mathrm{U} / \mathrm{ml}$.

\section{RÉSULTATS}

Les injections successives d'une même substance constituent une série. Les séries ont été réalisées dans l'ordre suivant : bradykinine, ocytocine, kallikréine, bradykinine et kallikréine.

(1) Phénergan R. Specia (Paris).

( $)$ Nobitocin. $\mathbf{S} \mathbf{R}$ Nobilis (Organon) Boxmeer.

(a) Padutine R. Bayer (Leverkusen). 


\section{A. - Première série de bradykinine} I à 4 ng.)

(I6 injections consécutives de 9 à $23 \mathrm{ng}, 1$ 'écart entre chaque dose variant de

La glande est très sensible à l'action de la bradykinine. A partir de I4 ng la pression intramammaire monte après Io à I5 secondes à une vitesse extrêmement rapide jusqu'à un niveau élevé (fig. I). D'autre part le retour de la courbe de pression vers le niveau de départ se fait d'une manière lente et en 2 phases en prenant un temps de 2 à 7 minutes (fig. I (I)). A la fin de cette série d'enregistrements le niveau de base de la pression a légèrement monté et l'amplitude des contractions provoquées par la bradykinine a diminué.

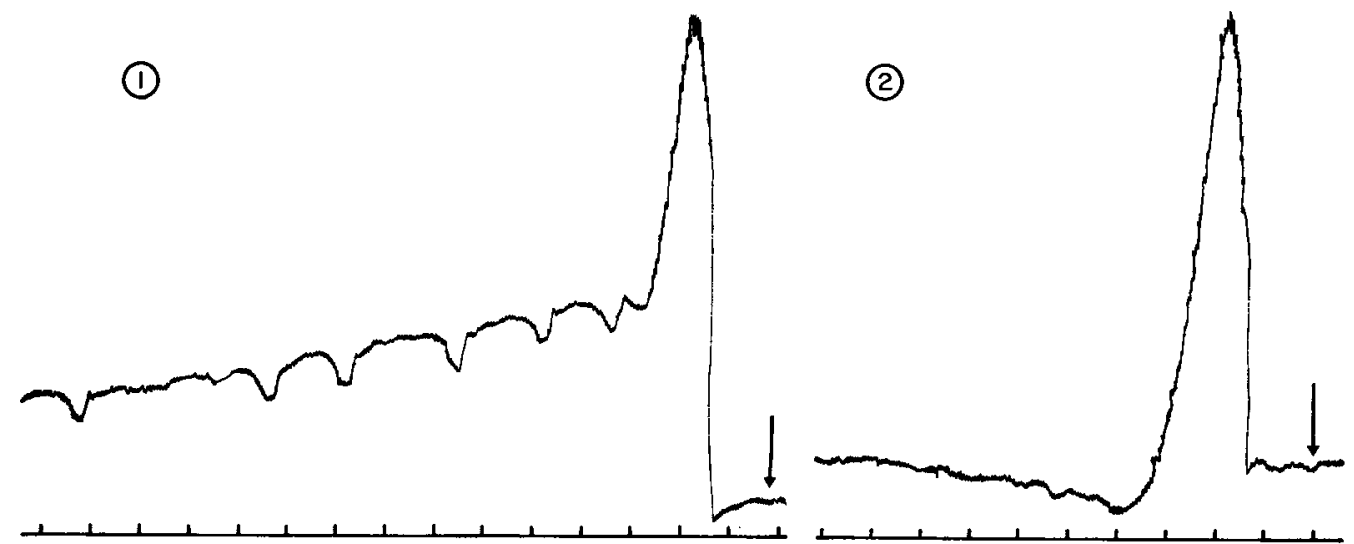

(3)

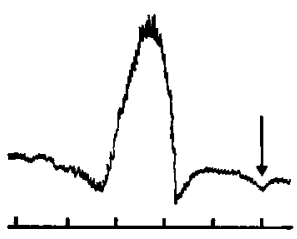

(4)

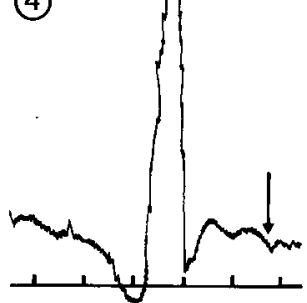

(5)

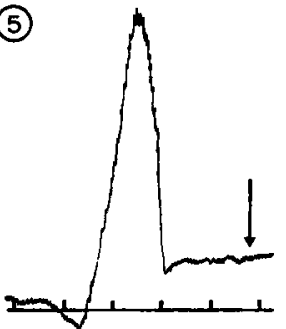

FIG. I. - Effets de la bradykinine, de l'ocytocine et de la kallikréine sur la pression intramammaire d'une brebis en lactation au cours des differentes phases de l'expérience $(\mathrm{I}, 2,3,4,5)$

$\downarrow$ moment de l'injection intrazrtérielle. Chaqua division de la ligne de base indique un espace de ro s. I. Bradykinine ( $19 \mathrm{ng}$ ). Descente très lente de la courbe de pression vers le niveau original.

2. Ocytocine $(190 \mu U)$. Descente rapide de la courbe et effet hypotensif postérieur.

3 , 4, 5. Ocytocine $(60 \mu \mathrm{U})$; kallikréine $(38 \mathrm{mU})$; bradykinine $(75 \mathrm{ng})$. Effets hypotensifs transitoires antérieurs et postérieurs.

\section{B. - Première série d'ocytocine}

(I I injections consécutives de 50 à I $90 \mu \mathrm{U}$, avec des écarts de Io à $20 \mu \mathrm{U}$ entre 50 et Iro $\mu \mathrm{U}$.)

L'ocytocine montre une activité à partir de $60 \mu \mathrm{U}$ après une latence de $\mathrm{I} 3$ à 24 secondes. Déjà la première injection d'ocytocine exerce un effet typique sur la 
pression intramammaire et ceci est confirmé par les injections suivantes. La descente de la PIM se déroule rapidement et il y a même une baisse passagère au-dessous du niveau originel de la pression (fig. I (2)). La durée de la courbe entière est beaucoup plus courte que dans la série $\mathrm{I}$. A partir de la $6^{\mathrm{e}}$ injection d'ocytocine $(80 \mu \mathrm{U})$ une baisse transitoire de la pression se manifeste immédiatement avant la montée de celle-ci (fig. I (3)). Cette baisse de pression antérieure est plus faible que la baisse postérieure. La courbe provoquée par la Io injection d'ocytocine (roo $\mu \mathrm{U}$ ) montre un aspect exceptionnel : la phase descendante, rapide au début, s'arrête brusquement et définitivement à mi-chemin. Ceci signifie que la pression de base s'est installée à un niveau supérieur. Ce niveau se maintient, abstraction faite de fluctuations moins importantes, durant la suite de l'expérience.

\section{C. - Première série de kallikréine}

(II injections consécutives de 30 à $500 \mathrm{mU}$, dont 9 doses entre 30 et $65 \mathrm{mU}$ ont des écarts de 2 à $\mathrm{I} 4 \mathrm{mU}$.)

La sensibilité de la glande est très bonne et des augmentations importantes de la pression sont constatées à partir de $30 \mathrm{mU}$. Les courbes de pression montrent les mêmes caractéristiques que celles provoquées par 1'ocytocine (latence de 8 à r6 secondes) : montée et descente rapides, courte durée de la reprise, effets hypotensifs transitoires aussi bien avant qu'après la courbe hypertensive, l'effet postérieur étant plus prononcé que l'effet antérieur (fig. I (4)).

\section{D. - Deuxième série de bradykinine}

(Io injections consécutives de $I_{5,5}$ à $82,5 \mathrm{ng}$, les écarts entre chaque dose ont varié de 8 à $25 \mathrm{ng}$.)

Les courbes montrent les mêmes caractéristiques que celles décrites pour 1'ocytocine et la kallikréine en ce qui concerne la durée, la latence, la vitesse de la phase descendante et les phénomènes hypotensifs antérieurs et postérieurs (fig. I (5)).

\section{E. - Deuxième série de kallikréine}

(34 injections de 4 à $200 \mathrm{mU}$, écart entre les doses 2 à $20 \mathrm{mU}$ pour 29 injections.) La glande réagit très bien à ces injections. Il est intéressant de constater que les chutes hypotensives antérieures se manifestent d'une manière beaucoup plus prononcée qu'auparavant, et il est clair que les 2 effets hypotensifs ne sont que des fractions d'une seule courbe hypotensive. Ainsi lors de l'injection d'une dose suffisamment faible de kallikréine, les 2 composants hypotensifs fusionnent en une seule courbe hypotensive tandis que l'effet hypertensif de la pression reste absent.

L'injection de doses plus élevées de kallikréine a provoqué des courbes que 1'on peut considérer comme la résultante d'effets hypotensifs et hypertensifs, dont le premier commence plus tôt et se termine plus tard que le second. Après l'injection, l'effet hypotensif se manifeste le premier mais il est interrompu brusquement et dominé temporairement par un effet de pression positive. Celui-ci montre un aspect normal de montée et de descente après quoi l'effet hypotensif entre dans sa phase 
terminale. En augmentant les doses administrées, l'effet hypertensif devient plus prononcé. Ainsi nous avons distingué dans cette série de kallikréine 4 types de courbes.
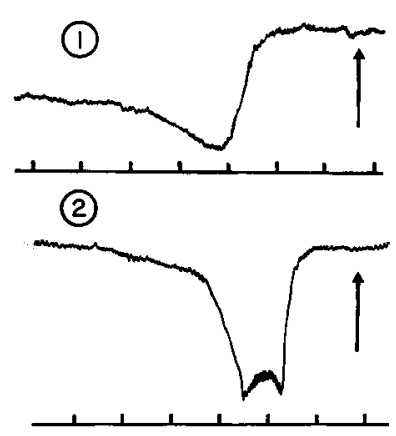

(3)

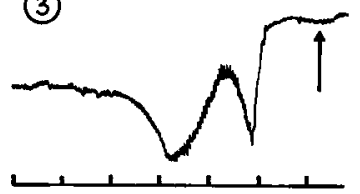

(4)
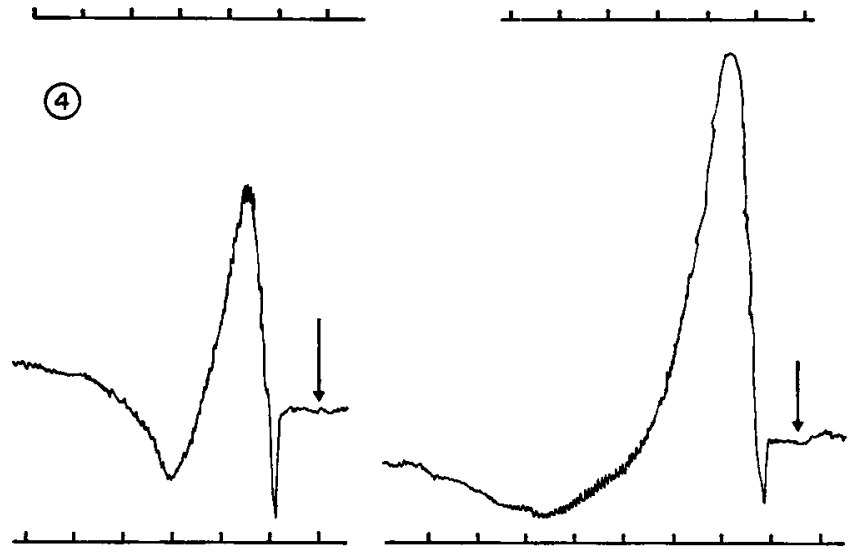

Fig. 2. - Effets variables de la kallikréine sur la pression intramammaire durant la série $V$

$\uparrow$ moment de l'injection intraartérielle

Chaque division de la ligne de base indique un espace de ro $\mathrm{s}$

$$
\begin{aligned}
& \text { I. Kallikréine (4 et }{ }_{5} \mathrm{mU} \text { ); courbes du type I } \\
& \text { 2. - (60 et } 40 \mathrm{mU}) \text {; - } 2 \\
& \begin{array}{llll}
\text { 3. } & -\quad(40 \text { et } \mathrm{roo} \mathrm{mU}) ; & - & 3 \\
4 . \quad(60 \text { et } 150 \mathrm{mU}) ; & - & 4
\end{array}
\end{aligned}
$$

Type 1. - Dans 3 cas nous avons obtenu des courbes à caractère uniquement hypotensif (fig. 2 (I)). Dans ces cas les doses injectées étaient faibles : 4, I5 et $30 \mathrm{mU}$.

Type 2. - Dans 8 cas nous avons constaté des courbes à caractère hypotensif prononcé. Cependant au moment où l'hypotension approche de sa valeur maximale, un effet hypertensif peu prononcé et de courte durée se manifeste (fig. 2 (2)). Les doses administrées étaient dans ces cas plus élevées que pour les courbes du type I $(6,10,20,40,50,55,60$ et $70 \mathrm{mU})$. 
Type 3. - Dans 6 cas nous avons obtenu des courbes analogues à celles du type 2, mais dont le composant hypertensif est plus prononcé, montant parfois jusqu'au niveau de pression précédant l'injection (fig. 2 (3)). Ces courbes étaient obtenues pour les doses suivantes : $30,30,4^{\circ}, 4^{\circ}, 5^{\circ}$ et $100 \mathrm{mU}$.

Type 4. - En injectant des doses plus élevées (6o, IIo, I30, I50, I75 et $200 \mathrm{mU}$ ) la courbe hypotensive débute de façon aiguë après une période de très courte latence ; elle atteint rapidement une valeur maximale, suivie alors d'une courbe hypertensive qui monte considérablement au-dessus du niveau originel (fig. 2 (4)).

Nos résultats montrent que l'effet hypotensif, absent au début de l'expérience (série I), se manifeste avec le temps de plus en plus nettement. Ainsi au cours de la première série de kallikréine, 1'injection de $50 \mathrm{mU}$ de kallikréine a causé des courbes hypertensives très prononcées, précédées et suivies d'un effet hypotensif faible. Dans la série $V$, la même dose de kallikréine a provoqué des courbes hypotensives très nettes avec un effet hypertensif faible (courbes du type 2).

Dans la série V (deuxième série kallikréine) les amplitudes des courbes hypertensives sont proportionnelles à la dose injectée; elles s'élèvent à o $\mathrm{mm}$ pour le type $1,7 \mathrm{~mm}$ pour le type $2,23 \mathrm{~mm}$ pour le type 3 et $80 \mathrm{~mm}$ pour le type 4 . Comme il résulte de la figure 2 les amplitudes des courbes hypotensives n'ont pu être mesurées, exception faite des courbes du type $I$. Le tableau $I$ indique que les périodes de latence des effets hypo- et hypertensifs sont plus brèves pour les doses élevées que pour les doses faibles.

TABI,EAU I

Périodes de latence (en secondes) entre l'injection et le début de la réaction du myoépithélium

\begin{tabular}{|c|c|c|c|}
\hline & \multicolumn{2}{|c|}{ Doses de kallikréine } & \\
\hline & $(4-60 \mathrm{mU})$ & $(110-200 \mathrm{mU})$ & \\
\hline Courbes hypotensives & $9,90 \pm 0,68$ & $5,87 \pm 1,19$ & $\mathrm{P}<0,05$ \\
\hline Courbes hypertensives . & $15,76 \pm 0,86$ & $8,37 \pm 1,10$ & $P<0,01$ \\
\hline
\end{tabular}

DISCUSSION ET CONCLUSION

WhitTLESTONE et ses collaborateurs (WhITrLESTONE, I950-I955; PHuLIPS, I968) ont effectué des enregistrements de la pression intramammaire chez des vaches. Des résultats obtenus, WhiTtLESTONE a avancé plusieurs arguments qui permettent de penser que les cellules myoépithéliales fusiformes situées longitudinalement autour des canaux galactophores, peuvent raccourcir et dilater ces canaux. Dans une de leurs expériences réalisée après une traite complète, 1a citerne de la glande mammaire d'une vache a été remplie avec une solution physiologique jusqu'à l'obtention d'une 
pression plus élevée que celle observée lors de l'éjection normale du lait. L'ocytocine, injectée ensuite, provoque une baisse de la pression intramammaire, suggérant l'apparition d'une dilatation des canaux galactophores.

Zars et MAzHBITZ (I965) ont injecté de l'ocytocine par voie sous-cutanée à 4 femmes souffrant de lactation persistante. L'ocytocine a provoqué une baisse de la pression intramammaire alors que cette hormone cause une augmentation au cours de la lactation normale. Ces résultats peuvent s'interpréter par la contraction du myoépithélium entourant les canaux microscopiques. Les auteurs ont d'ailleurs suggéré que cet effet était dû à un développement mal équilibré entre le myoépithélium des alvéoles et celui des canaux dans la glande pathologique.

Dans le même ordre d'idée, LINZELL, (1955) a également observé in vivo à l'aide d'un microscope les contractions des alvéoles et des canaux des glandes mammaires de rats, de souris, de cobayes et de lapines. L'ocytocine administrée à la surface du tissu mammaire a provoqué la contraction des alvéoles, le raccourcissement et la dilatation des canaux galactophores. Cette dilatation pourrait être considérée comme un phénomène passif causé par l'expulsion du lait des alvéoles. Cependant, chez les animaux ayant des alvéoles dégénérées et ne contenant plus de lait, l'ocytocine a conduit également au raccourcissement et à la dilatation des canatux. Utilisant la même technique chez des souris, LEVITSKaYA (I955) a observé la dilatation de ces canaux galactophores et la contraction des alvéoles après administration d'acétylcholine ou d'hormone posthypophysaire. Elle souligne que la dilatation des canaux commence un peu avant la contraction des alvéoles, ce qui témoigne en faveur d'une influence directe de l'hormone sur le myoépithélium des canaux au lieu d'une distension passive de ceux-ci.

A la lumière de toutes ces observations il semble que, dans nos expériences, les courbes hypertensives sont causées par la contraction du myoépithélium situé autour des alvéoles tandis que les effets hypotensifs sont provoqués par la contraction des cellules myoépithéliales placées longitudinalement autour des canaux galactophores. Au début de notre expérience le myoépithélium des alvéoles a réagi d'une manière puissante aux 3 substances examinées. Par contre, le myoépithélium des canaux galactophores ne s'est apparemment pas contracté pendant la première série de bradykinine (I), et les canaux se sont comportés comme des valves permettant la sortie facile du lait des alvéoles mais rendant son retour difficile. Ceci a conduit à une augmentation progressive de la pression citernale. La première injection d'ocytocine (série II) a causé une certaine relaxation active des canaux galactophores se manifestant par un retour facile au niveau de base de la courbe de pression accompagnée d'un effet hypotensif transitoire. Ceci a été confirmé lors des injections suivantes, l'ocytocine donnant lieu même de temps en temps à une légère dilatation active des canaux avant la contraction alvéolaire. Cependant, l'interruption brusque et prématurée de la phase descendante de la courbe de pression après la Io injection d'ocytocine indique à nouveau l'apparition d'une contraction spasmodique des petits canaux galactophores. La kallikréine (série III) et la bradykinine (série IV) ont provoqué également la distension des canaux, celle-ci commençant plus tôt et durant plus longtemps que la contraction alvéolaire. Au cours de la série $\mathrm{V}$ la sensibilité du myoépithélium des canaux à l'action de la kallikréine a été très élevée, les doses efficaces sur les canaux étant plus faibles que celles agissant sur la contraction des alvéoles. Dans un cas la dose de $4 \mathrm{mU}$ a donné lieu à la disten- 
sion des canaux galactophores sans influencer le myoépithélium des alvéoles. Des doses plus élevées ont provoqué la contraction des 2 systèmes mais les réactions des canaux se sont toujours manifestées les premières et ont été plus prolongées. Des doses supérieures à Ioo $\mathrm{mU}$ ont stimulé fortement les 2 systèmes contractiles. Par conséquent les effets sur les 2 systèmes apparaissent directement proportionnels aux doses administrées.

Ainsi chacune des 3 substances examinées cause la contraction du myoépithélium des canaux galactophores : l'ocytocine dans la série II, la bradykinine đans la série IV et la kallikréine dans les séries III et V. On peut alors se demander pourquoi la sensibilité du myoépithélium des canaux a augmenté graduellement au cours de l'expérience. Ce phénomène pourrait être attribué éventuellement au reflux incomplet du lait vers les alvéoles au début de l'expérience et à l'augmentation progressive de la pression intramammaire. L'augmentation de la pression faciliterait ensuite le passage du lait de la citerne vers les alvéoles pendant la relaxation des canaux galactophores et de cette manière les effets hypotensifs se manifesteraient d'une manière plus prononcée dans la citerne. Dans les expériences de WHI'TrLIESTONE (I950) l'ocytocine a également provoqué une baisse de la pression après le remplissage de la citerne avec une solution physiologique sous pression.

Il est possible que, grâce à la tendance qu'ont les canaux à montrer une certaine tonicité, nous avons eu la possibilité d'observer dans cette expérience leur dilatation après l'administration d'ocytocine et des kinines. Il faut tout de même s'interroger sur:le fait que nous n'avons pu répéter nos observations au cours de 3 expériences ultérieures: sur la même brebis, ni sur les autres animaux étudiés. Il se pourrait que la pression dans la citerne et le tonus des canaux galactophores se trouvaient dans l'expérience décrite dans des conditions optimales pour la mise en évidence de leurs contractions. Il est logique de penser que le degré de sensibilité du myoépithélium autour des canaux est parallèle à celui du myoépithélium autour des alvéoles. D'autre part la dilatation des canaux facilite l'expulsion du lait hors des alvéoles ce qui permet à des doses faibles d'hormone d'être effectives sur le myoépithélium alvéolaire. Tout ceci démontre que nous ne comprenons pas encore complètement la coordination des activités des 2 groupes de cellules myoépithéliales.

Nos résultats obtenus chez la Brebis sont en accord avec les observations des auteurs cités plus haut chez d'autres espèces. Le fait que les effets hypotensifs débutent plus précocement que les modifications hypertensives, confirme les constatations de Levirskaya (I955) qui remarque chez la Souris que la dilatation des petits canaux précède la contraction des alvéoles. Nos expériences ont en outre démontré que les kinines et les kallikréines peuvent également dilater les canaux.

Rę̧u pour publication en janvier 1973.

\section{REMERCIEMENTS}

Cette recherche a été financée par l'Institut pour l'Encouragement de la Recherche scientifique dans l'Industrie et l'Agriculture (I. R. S. I. A.). 


\section{SUMLMARY}

\section{VARYING EFFECTS INDUCED BY OXYTOCIN AND KININS ON INTRAMAMMARY PRESSURE ON A LACTATING EWE}

Intramammary pressure was recorded in numerous experiments carried out on lactating ewes and goats. The animals were fixed on their back under general anaesthesia. The action of oxytocin, bradykinin and kallikrein, injected directly in the artery of the mammary gland, was studied. In I sole experiment on I ewe, the 3 substances induced either increases or inhibitions of the pressure in the cistern. The hypertensive effects are attributed to the activity of the myoepithelial cells situated around the alveoli inducing the expulsion of milk. The hypotensive effects would be produced by contraction of myoepithelial cells situated longitudinally on the fine milkducts inducing shortening and dilatation of these ducts. During the second half of the experiment the myoepithelium around the ducts appeared to be more sensitive to the action of the substances studied than the myoepithelium of the alveoli. Dilatation of the ducts was induced by smaller doses, occurred after a shorter latency and was maintained longer than contraction of the alveoli.

\section{RÉFÉRENCES BIBLIOGRAPHIQUES}

Levitskaya E. S., r955. Études in vivo sur le fonctionnement de l'appareil de l'éjection de la glande mammaire chez les souris blanches. Tr. Inst. fiziol. im. I. P. Pavlova, 4, 58-62 (russe).

LINzELL J. L., r952. The silver staining of myoepithelial cells, particularly in the mammary gland, and their relation to the ejection of milk. J. Anat., 86, 49-57.

Linzel. J. L., I955. Some observations on the contractile tissue of the mammary glands. J. Physiol., 180, $257-267$.

Peeters G., Houvenaghel A., Verbeke R., Van Sichem-Reynaert R., I972. Effects of bradykinin and kallikrein injected into the udder artery of sheep and goats. Arch. int. Pharmacodyn., 198, 397-414.

Phillips D. S. M., rg68. Changes in intramammary pressure and distribution of milk in the udder due to withdrawal at varying intervals after establishment of the milk ejection response. $N . Z$. $J l$. agric. Res., 11, 47-58.

RicharDSON K. C., I949. Contractile tissues in the mammary gland, with special reference to myoepithelium in the goat. Proc. Roy. Soc. London, 136, 30-45.

Whirtesston W. G., r950. Nature of the milk-ejection process. Nature, Lond., 166, 994.

Whittleston W. G., r955. Intramammary pressure changes in the lactating cow. I. Changes during the milking process. J. dairy Res., 22, 290-294.

ZAks M. G., Mazhbitz I. A., I965. La réaction paradoxale de la glande mammaire sur l'ocytocine chez des femmes avec lactation persistante. Byull. éksp. Biol. Med., 59, 53-55 (russe). 